\title{
Uniqueness of meromorphic functions whose differential polynomials share a nonconstant polynomial
}

\author{
Dilip Chandra Pramanik and Jayanta Roy \\ Department of Mathematics, University of North Bengal, Raja Rammohunpur, Darjeeling-734013, West Bengal, India \\ Received: 24 August 2018, Accepted: 4 January 2019 \\ Published online: 21 June 2019.
}

Abstract: In this paper, we study the uniqueness of meromorphic functions whose differential polynomials share a nonconstant polynomial generalizing some earlier results.

Keywords: Meromorphic function, small function, uniqueness, sharing value, differential polynomial.

\section{Introduction}

In this paper, by meromorphic function we shall always mean a meromorphic function in the complex plane. We adopt the standard notations in the Nevanlinna Theory of meromorphic functions as explained in $[4,9,18]$. It will be convenient to let $E$ denote any set of positive real numbers of finite linear measure, not necessarily the same at each occurrence.

For any nonconstant meromorphic function $f(z)$, we denote by $S(r, f)$ any quantity satisfying $S(r, f)=\circ(T(r, f))$ as $r \rightarrow \infty, r \notin E$. A meromorphic function $a(z)$ is said to be small with respect to $f(z)$ if $T(r, a)=S(r, f)$. We denote by $S(f)$ the collection of all small functions with respect to $f$. Clearly $\mathbb{C} \cup\{\infty\} \in S(f)$ and $S(f)$ is a field over the set of complex numbers.

For any two nonconstant meromorphic functions $f$ and $g$, and $a \in S(f) \cap S(g)$, we say that $f$ and $g$ share " $a$ " IM(CM) provided that $f-a$ and $g-a$ have the same zeros ignoring(counting) multiplicities.

The following theorem in the value distribution theory is well known [3,15].

Theorem 1. Let $f(z)$ be a transcendental meromorphic function, $n \geq 1$ a positive integer. Then $f^{n} f^{\prime}=1$ has infinitely many solutions.

Fang and Hua [12], Yang and Hua [20] obtained a unicity theorem respectively to the above theorem.

Theorem 2. Let $f(z)$ and $g(z)$ be two nonconstant entire (meromorphic) functions, $n \geq 6(n \geq 11)$ be a positive integer. If $f^{n} f^{\prime}$ and $g^{n} g^{\prime}$ share $1 C M$ then either $f(z)=c_{1} \exp (c z), g(z)=c_{2} \exp (-c z)$, where $c_{1}, c_{2}$ and $c$ are three constant satisfying $4\left(c_{1} c_{2}\right)^{n+1} c^{2}=-1$, or $f(z) \equiv \operatorname{tg}(z)$ for a constant $t$ such that $t^{n+1}=1$.

Theorem 3. Let $f(z)$ and $g(z)$ be two nonconstant entire functions, let $n, k$ be two positive integers with $n>2 k+4$. If $\left(f^{n}(z)\right)^{(k)}$ and $\left(g^{n}(z)\right)^{(k)}$ share $1 C M$ then either $f(z)=c_{1} \exp (c z), g(z)=c_{2} \exp (-c z)$, where $c_{1}$, $c_{2}$ and $c$ are three constant satisfying $(-1)^{k}\left(c_{1} c_{2}\right)^{n}(n c)^{2 k}=1$ or $f(z) \equiv \operatorname{tg}(z)$ for a constant $t$ such that $t^{n}=1$. 
Theorem 4. Let $f(z)$ and $g(z)$ be two nonconstant entire functions and let $n, k$ be two positive integers with $n>2 k+8$. If $\left(f^{n}(z)(f(z)-1)\right)^{(k)}$ and $\left(g^{n}(z)(g(z)-1)\right)^{(k)}$ share 1 CM then $f(z) \equiv g(z)$.

Then Fang and Qiu [10] considered the fixed point sharing uniqueness problem and obtained the following theorem.

Theorem 5. Let $f(z)$ and $g(z)$ be two nonconstant entire functions and $n \geq 6$ be a positive integer. If $f^{n}(z) f^{\prime}(z)$ and $g^{n}(z) g^{\prime}(z)$ share $z C M$, then either $f(z)=c_{1} e^{c z^{2}}, g(z)=c_{2} e^{-c z^{2}}$, where $c_{1}, c_{2}$ and $c$ are three constant satisfying $4\left(c_{1} c_{2}\right)^{n+1} c^{2}=-1$ or $f(z) \equiv \operatorname{tg}(z)$ for a constant $t$ such that $t^{n+1}=1$.

Later in 2004, Lin and Yi [16] proved the following theorem:

Theorem 6. Let $f$ and $g$ be two nonconstant entire functions and $n \geq 7$ be a positive integer. If $f^{n}(f-1) f^{\prime}$ and $g^{n}(g-1) g^{\prime}$ share z $C M$, then $f \equiv g$.

Zhang [6] extended the above two theorems and got the following results:

Theorem 7. Let $f(z)$ and $g(z)$ be two nonconstant entire functions and let $n, k$ be two positive integers with $n>2 k+4$. If $\left(f^{n}\right)^{(k)}$ and $\left(g^{n}\right)^{(k)}$ share $z$ CM, then either

(1) $k=1, f(z)=c_{1} e^{c z^{2}}, g(z)=c_{2} e^{-c z^{2}}$, where $c_{1}, c_{2}$ and $c$ are three constant satisfying $4\left(c_{1} c_{2}\right)^{n}(n c)^{2}=-1$, or

(2) $f(z) \equiv \operatorname{tg}(z)$ for a constant $t$ such that $t^{n}=1$.

Theorem 8. Let $f$ and $g$ be two nonconstant entire functions and let $n$, $k$ be two positive integers with $n>2 k+6$. If $\left(f^{n}(f-1)\right)^{(k)}$ and $\left(g^{n}(g-1)\right)^{(k)}$ share $z C M$, then $f \equiv g$.

Regarding Theorems 1.7 - 1.8, Xu et al [7] considered the case of meromorphic functions. They proved.

Theorem 9. Let $f$ and $g$ be two nonconstant meromorphic functions and let $n, k$ be two positive integers with $n>3 k+10$. If $\left(f^{n}\right)^{(k)}$ and $\left(g^{n}\right)^{(k)}$ share $z C M, f$ and $g$ share $\infty I M$, then either $f(z)=c_{1} e^{c z^{2}}, g(z)=c_{2} e^{-c z^{2}}$, where $c_{1}, c_{2}$ and $c$ are three constant satisfying $4\left(c_{1} c_{2}\right)^{n}(n c)^{2}=-1$ or $f(z) \equiv \operatorname{tg}(z)$ for a constant $t$ such that $t^{n}=1$.

Theorem 10. Let $n, k$ be two positive integers with $n>3 k+12$, and $f$ and $g$ be two nonconstant meromorphic functions satisfying $\Theta(\infty, f)>\frac{2}{n}$. If $\left(f^{n}(f-1)\right)^{(k)}$ and $\left(g^{n}(g-1)\right)^{(k)}$ share $z C M$, $f$ and $g$ share $\infty I M$, then $f \equiv g$.

In view of above theorems, Sahoo [13] obtained the following result in 2010 for some more general nonlinear differential polynomial.

Theorem 11. Let $f$ and $g$ be two nonconstant meromorphic functions and let $n, k$ and $m$ be three positive integers with $n>9 k+4 m+13$. Let $P(w)=a_{m} w^{m}+a_{m-1} w^{m-1}+\cdots+a_{1} w+a_{0}$ where $a_{0} \neq 0, a_{1}, \cdots, a_{m} \neq 0$ are complex constants. If $\left(f^{n} P(f)\right)^{(k)}$ and $\left(g^{n} P(g)\right)^{(k)}$ share $z I M, f$ and $g$ share $\infty$ IM. Then $f=t g$ for a constant $t$ such that $t^{d}=1$, where $d=\operatorname{gcd}(n+m, n+m-1, \ldots, n+m-i, \ldots, n+1, n), a_{m-i} \neq 0$ for some $i \in\{0,1, \ldots, m\} ;$ or $f$ and $g$ satisfy the algebraic equation $R(f, g)=0$ where $R(f, g)$ is given by

$$
R(f, g)=f^{n}\left(a_{m} f^{m}+\ldots+a_{1} f+a_{0}\right)-g^{n}\left(a_{m} g^{m}+\ldots+a_{1} g+a_{0}\right) .
$$

It is natural question to ask what happen if sharing fixed point in above theorem is replaced by sharing a nonconstant polynomial.

Keeping in mind the above question X. B. Zhang and J. F. Xu [19] obtained the following result:

Theorem 12. Let $f$ and $g$ be two nonconstant meromorphic functions, $p(z)$ be a nonconstant polynomial of degree $\operatorname{deg}(p)=l \leq 5, n, k$ and $m$ be three positive integers with $n>3 k+m+7$. Let $P(w)=a_{m} w^{m}+a_{m-1} w^{m-1}+\cdots+a_{1} w+a_{0}$ be a nonzero polynomial. If $\left(f^{n} P(f)\right)^{(k)}$ and $\left(g^{n} P(g)\right)^{(k)}$ share $p C M, f$ and $g$ share $\infty$ IM, then one of the following two cases holds: 
(1) $f=$ tg for a constant $t$ such that $t^{d}=1$, where $d=\operatorname{gcd}(n+m, n+m-1, \ldots, n+m-i, \ldots, n+1, n)$, $a_{m-i} \neq 0$ for some $i \in\{0,1, \ldots, m\}$;

(2) $f$ and $g$ satisfy the algebraic equation $R(f, g)=0$ where $R(f, g)$ is given by (1).

(3) $P(z)$ is reduced to a nonzero monomial, namely $P(z)=a_{i} z^{i} \not \equiv 0$ for some $i \in\{0,1, \ldots, m\}$; if $p(z)$ is not a constant, then $f(z)=c_{1} e^{c Q(z)}, g(z)=c_{2} e^{-c Q(z)}$, where $Q(z)=\int_{0}^{z} p(z) d z, c_{1}, c_{2}$ and $c$ are three constants satisfying $a_{i}^{2}\left(c_{1} c_{2}\right)^{n+i}((n+i) c)^{2}=-1$, if $p(z)$ is a constant $b$, then $f(z)=c_{3} e^{c z}, g(z)=c_{4} e^{-c z}, c_{3}, c_{4}$ and c are three constants satisfying $(-1)^{k} a_{i}^{2}\left(c_{3} c_{4}\right)^{n+i}((n+i) c)^{2 k}=b^{2}$.

In 2016, Sahoo et al [14] removed the restriction on the degree of the polynomial $p(z)$ and proved the following theorems:

Theorem 13. Let $f$ and $g$ be two transcendental meromorphic functions, $p(z)$ be a nonconstant polynomial of degree $l$, and let $n(\geq 1), k(\geq 1)$ and $m(\geq 0)$ be three integers with $n>\max \{3 k+m+6, k+2 l\}$. In addition we suppose that either $k, l$ are co-prime or $k>l$ when $l \geq 2$. Let $P(w)$ be defined as in the above theorem. If $\left(f^{n} P(f)\right)^{(k)}$ and $\left(g^{n} P(g)\right)^{(k)}$ share $p(z) C M ; f$ and $g$ share $\infty$ IM, then the following conclusions hold:

(i) If $P(w)=a_{m} w^{m}+a_{m-1} w^{m-1}+\cdots+a_{1} w+a_{0}$ is not a monomial, then either $f=t g$ for a constant that satisfies $t^{d}=$ 1 , where $d=\operatorname{gcd}(n+m, n+m-1, \ldots, n+m-i, \ldots, n+1, n), a_{m-i} \neq 0$ for some $i \in\{0,1, \ldots, m\}$; or $f$ and $g$ satisfy the algebraic equation $R(f, g)=0$ where $R(f, g)$ is given by (1). In particular $m=1$ and $\Theta(\infty, f)+\Theta(\infty, g)>\frac{4}{n}$, then $f=g$.

(ii) When $P(w)=a_{m} w^{m}$, or $P(w)=c_{0}$, then either $f=$ tg for some $t$ such that $t^{n+m^{*}}=1$, or $f(z)=b_{1} e^{b Q(z)}, g(z)=$ $b_{2} e^{-b Q(z)}$, where $Q(z)$ is a polynomial without constant such that $Q^{\prime}(z)=p(z), b_{1}, b_{2}$ and $b$ are three constants satisfying $a_{m}^{2}\left(b_{1} b_{2}\right)^{n+m}((n+m) b)^{2}=-1$ or $c_{0}^{2}\left(b_{1} b_{2}\right)^{n}(n b)^{2}=-1$, where $m^{*}$ is same as in Lemma 13.

Theorem 14. Let $f$ and $g$ be two transcendental meromorphic functions, $p(z)$ be a nonconstant polynomial of degree $l$, and let $n(\geq 1), k(\geq 1)$ and $m(\geq 0)$ be three integers with $n>\max \{9 k+4 m+11, k+2 l\}$. In addition we suppose that either $k, l$ are co-prime or $k>l$ when $l \geq 2$. Let $P(w)$ be defined as in the above theorem. If $\left(f^{n} P(f)\right)^{(k)}$ and $\left(g^{n} P(g)\right)^{(k)}$ share $p(z) I M ; f$ and $g$ share $\infty$ IM, then the conclusion of Theorem 13 hold.

In this paper we investigate on the above theorem to remove sharing $\infty$ IM and obtain the following results:

Theorem 15. Let $f$ and $g$ be two transcendental meromorphic functions with $\sigma(f)<\infty$, whose zeros and poles are of multiplicity at least $s$, where $s$ is positive integer. Let $p(z)$ is a polynomial of degree $l$ and

$$
P(w)=a_{m} w^{m}+a_{m-1} w^{m-1}+\cdots+a_{1} w+a_{0},
$$

where $a_{0}, a_{1}, \cdots, a_{m}$ are constants, where $n(\geq 1), k(\geq 1), m(\geq 0)$ be three integers and $k>l$ when $l \geq 2$ satisfying

$$
n>\max \left\{2 k+2 l, \frac{3 k+8}{s}+m, 2 k(\sigma(f)-1)-(m+2 l)\right\} \text {. }
$$

If $\left(f^{n} P(f)\right)^{(k)}$ and $\left(g^{n} P(g)\right)^{(k)}$ share $p(z) C M$, then one of the following holds:

(i) If $P(w)$ is not a monomial and (3) hold.Then either $f=$ tg for some $t$ such that $t^{d}=1$, where $d=\operatorname{gcd}(n+m, n+m-$ $1, \ldots, n+m-i, \ldots, n+1, n), a_{m-i} \neq 0$ for some $i \in\{0,1, \ldots, m\}$; or $f$ and $g$ satisfy the algebraic equation $R(f, g)=0$ where $R(f, g)$ is given by $(1)$.

(ii) $P(w)=a_{m} w^{m}$, or $P(w)=c_{0}$, then either $f=$ tg for a constant $t$ such that $t^{n+m^{*}}=1$, or $f(z)=b_{1} e^{b Q(z)}, g(z)=$ $b_{2} e^{-b Q(z)}$, where $Q(z)$ is a polynomial without constant such that $Q^{\prime}(z)=p(z), b_{1}, b_{2}$ and $b$ are three constants satisfying $a_{m}^{2}\left(b_{1} b_{2}\right)^{n+m}((n+m) b)^{2}=-1$ or $c_{0}^{2}\left(b_{1} b_{2}\right)^{n}(n b)^{2}=-1$, where $m^{*}$ is same as in Lemma 13. 
Theorem 16. Let $f$ and $g$ be two transcendental meromorphic functions with $\sigma(f)<\infty$, whose zeros and poles are of multiplicity at least $s$, where $s$ is positive integer. Let $p(z)$ is a polynomial of degree l and $P(w)=a_{m} w^{m}+a_{m-1} w^{m-1}+\cdots$ $\cdot+a_{1} w+a_{0}$ be a nonzero polynomial and $n(\geq 1), k(\geq 1), m(\geq 0)$ be three integers and $k>l$ when $l \geq 2$ satisfying

$$
n>\max \left\{2 k+2 l, \frac{9 k+14}{s}+4 m, 2 k(\sigma(f)-1)-(m+2 l)\right\} .
$$

If $\left(f^{n} P(f)\right)^{(k)}$ and $\left(g^{n} P(g)\right)^{(k)}$ share $p(z)$ IM, then the conclusions of Theorem 15 hold.

\section{Lemmas}

Let $F_{1}$ and $G_{1}$ be nonconstant meromorphic functions defined in a complex plane $\mathbb{C}$. We denote by $H$ the following function:

$$
H=\left(\frac{F_{1}^{(2)}}{F_{1}^{(1)}}-2 \frac{F_{1}^{(1)}}{F_{1}-1}\right)-\left(\frac{G_{1}^{(2)}}{G_{1}^{(1)}}-2 \frac{G_{1}^{(1)}}{G_{1}-1}\right) .
$$

Lemma 1. [2] Let $f(z)$ be a nonconstant meromorphic function and let $a_{0}(z), a_{1}(z), \ldots .$. $a_{n}(z)(\not \equiv 0)$ be a small function with respect to $f$. Then

$$
T\left(r, a_{n} f^{n}+a_{n-1} f^{n-1}+\ldots+a_{0}\right)=n T(r, f)+S(r, f) .
$$

Lemma 2. [4] Let $f(z)$ be a nonconstant meromorphic function in the complex plane. If the order of $f$ is finite, then

$$
m\left(r, \frac{f^{\prime}}{f}\right)=O(\log r), r \rightarrow \infty .
$$

Lemma 3. [4] Let $h(z)$ be a nonconstant entire function and let $f(z)=e^{h(z)}$. Let $\lambda$ and $\mu$ be the order and lower order of $h(z)$, respectively. We have

(i) If $\mu<\infty$, then $\mu$ is a positive integer, $h(z)$ is a polynomial of degree $\mu$, and $\lambda=\mu$.

(ii) If $\mu<\infty$, then $h(z)$ is transcendental and $\lambda=\mu$.

Lemma 4. [17] Let $f(z)$ be a nonconstant meromorphic function and $s, k$ be two positive integers. Then

$$
\begin{gathered}
N_{s}\left(r, \frac{1}{f^{(k)}}\right) \leq T\left(r, f^{(k)}\right)-T(r, f)+N_{s+k}\left(r, \frac{1}{f}\right)+S(r, f) . \\
N_{s}\left(r, \frac{1}{f^{(k)}}\right) \leq k \bar{N}(r, f)+N_{s+k}\left(r, \frac{1}{f}\right)+S(r, f) .
\end{gathered}
$$

Lemma 5. [2] Let $f(z)$ be a nonconstant meromorphic function and let $k$ be a positive integer. Suppose that $f^{(k)} \not \equiv 0$, then

$$
N\left(r, \frac{1}{f^{(k)}}\right) \leq k \bar{N}(r, f)+N\left(r, \frac{1}{f}\right)+S(r, f)
$$

Lemma 6. [5] If $N\left(r, 0 ; f^{(k)} / f \neq 0\right)$ denotes the counting function of those zeros of $f^{(k)}$ that are not the zeros of $f$, where a is zero of $f^{(k)}$ is counted according to its multiplicity, then

$$
N\left(r, \frac{1}{f^{(k)}} / f \neq 0\right) \leq k \bar{N}(r, f)+N\left(r, \frac{1}{f} \mid<k\right)+k \bar{N}\left(r, \frac{1}{f} \mid \geq k\right)+S(r, f) .
$$


Lemma 7. Let $f$ and $g$ be two nonconstant transcendental meromorphic functions whose zeros and poles are of multiplicity at least $s$, where $s$ is positive integers. Let $P(w)=a_{m} w^{m}+a_{m-1} w^{m-1}+\cdots+a_{1} w+a_{0}$, where $a_{0}(\neq 0), a_{1}, \cdots, a_{m}(\neq 0)$ are constants, and let $n(\geq 1), k(\geq 1), m(\geq 0)$ are integers and $p(z)$ is a polynomial of degree $l$. If

$$
\lambda>\frac{1}{s}+\frac{m+n}{(m+n) s+2 k}
$$

where $\lambda$ is the number of distinct roots of $P(w)=0$ then

$$
\left(f^{n} P(f)\right)^{(k)}\left(g^{n} P(g)\right)^{(k)} \neq p^{2}(z)
$$

Proof.

$$
\left(f^{n} P(f)\right)^{(k)}\left(g^{n} P(g)\right)^{(k)}=p^{2}(z)
$$

Let $P(z)=a_{m}\left(z-d_{1}\right)^{l_{1}} \cdot\left(z-d_{2}\right)^{l_{2}} \cdots\left(z-d_{\lambda}\right)^{l_{\lambda}}$, where $\sum_{j=1}^{\lambda} l_{j}=m, 1 \leq \lambda \leq m, d_{i} \neq d_{j}, i \neq j$ and $1 \leq i, j \leq \lambda, d_{j}$ 's are nonzero constants and $l_{j}$ 's are positive integers, $j=1,2, . ., \lambda$. Let $z_{0} \notin\{z: p(z)=0\}$ be a zero of $f$ with multiplicity $p_{0}(\geq s)$. Then $z_{0}$ is a pole of $g$ with multiplicity $q_{0}(\geq s)$ say. From (4) we get, $n p_{0}-k=(m+n) q_{0}+k$ so, $n p_{0}-k \geq$ $(m+n) s+k$ and $p_{0} \geq \frac{(m+n) s+2 k}{n}$. Let $z_{1} \notin\{z: p(z)=0\}$ be a zero of $P(f)$ of order $p_{1}$ and a zero of $f-d_{i}$ of order $q_{i}$ for some $i=1,2, \ldots \lambda$. Then $p_{1}=l_{i} q_{i}$ for some $i=1,2, \ldots \lambda$, and $z_{1}$ is a pole of $g$ with multiplicity $\bar{q}(\geq s)$ say, so from (4) we get

$$
\begin{aligned}
q_{i} l_{i}-k & =(n+m) \bar{q}+k \\
& \geq(n+m) s+k \\
\text { i.e., } q_{i} & \geq \frac{(n+m) s+2 k}{l_{i}}
\end{aligned}
$$

for $i=1,2, . ., \lambda$. Let $z_{2} \notin\{z: p(z)=0\}$ be a zero of $\left(f^{n} P(f)\right)^{(k)}$ of order $p_{2}$ but not a zero of $f^{n} P(f)$. Then from (4) $z_{2}$ is a pole of $g$ of order $\xi(\geq s)$. Then

$$
p_{2}=(n+m) \xi+k \geq(n+m) s+k .
$$

Suppose that $z_{3} \notin\{z: p(z)=0\}$ be a pole of $f$ then from (4) $z_{3}$ is a zero of $\left(g^{n} P(g)\right)$ or a zero of $\left(g^{n} P(g)\right)^{(k)}$. Therefore

$$
\bar{N}(r, f) \leq \bar{N}\left(r, \frac{1}{g}\right)+\sum_{j=1}^{\lambda} \bar{N}\left(r, \frac{1}{g-d_{i}}\right)+\bar{N}\left(r, 0 ; B^{k} / B \neq 0\right)+S(r, g),
$$

where $\bar{N}\left(r, 0 ; B^{(k)} / B \neq 0\right)$ denotes the reduced counting function of those zeros of $B^{(k)}$ that are not the zeros of $B$ and $B=g^{n} P(g)$. Now by Lemma 6 we have

$$
\begin{aligned}
\bar{N}\left(r, 0 ; B^{(k)} / B \neq 0\right) & \leq \frac{1}{(n+m) s+k} N\left(r, \frac{1}{B^{(k)}} / B \neq 0\right) \\
& \leq \frac{1}{(n+m) s+k}\left\{k \bar{N}(r, B)+N\left(r, \frac{1}{B} \mid<k\right)+k \bar{N}\left(r, \frac{1}{B} \mid \geq k\right)\right\} \\
& \leq \frac{k}{(n+m) s+k}\left\{\bar{N}(r, B)+N_{k}\left(r, \frac{1}{B}\right)\right\} \\
& \leq \frac{k}{(n+m) s+k}\left\{\bar{N}\left(r, \frac{1}{g}\right)+\sum_{j=1}^{\lambda} \bar{N}\left(r, \frac{1}{g-d_{i}}\right)+\bar{N}(r, g)\right\}
\end{aligned}
$$


So,

$$
\begin{aligned}
\bar{N}(r, f) & \leq\left(1+\frac{k}{(n+m) s+k}\right)\left(\frac{n}{(m+n) s+2 k}+\frac{m}{(m+n) s+2 k}\right) T(r, g) \\
& +\frac{k}{((n+m) s+k) s} T(r, g)+S(r, f)+S(r, g) \\
& \leq \frac{1}{s} T(r, g)+S(r, f)+S(r, g) .
\end{aligned}
$$

By second fundamental theorem

$$
\begin{aligned}
\lambda T(r, f) & \leq \bar{N}(r, f)+\bar{N}\left(r, \frac{1}{f}\right)+\sum_{j=1}^{\lambda} \bar{N}\left(r, \frac{1}{f-d_{i}}\right)+S(r, f) \\
& \leq\left(\frac{n}{(m+n) s+2 k}+\frac{m}{(m+n) s+2 k}\right) T(r, f)+\frac{1}{s} T(r, g)+S(r, f)+S(r, g) .
\end{aligned}
$$

Similarly,

$$
\lambda T(r, g) \leq\left(\frac{n}{(m+n) s+2 k}+\frac{m}{(m+n) s+2 k}\right) T(r, g)+\frac{1}{s} T(r, f)+S(r, f)+S(r, g) .
$$

Adding above two inequality we get

$$
\left\{\lambda-\frac{1}{s}-\frac{m+n}{(m+n) s+2 k}\right\}(T(r, f)+T(r, g)) \leq S(r, f)+S(r, g)
$$

which contradict given assumption. This completes the proof of the Lemma.

Lemma 8. Let $f$ and $g$ be two nonconstant meromorphic functions with $\sigma(f)<\infty, p(z)$ be nonconstant polynomial of degree $l$ and $n, k, m$ be three positive integer with $n>\max \{2 k+2 l, 2 k(\sigma(f)-1)-(m+2 l)\}$. In addition we assume $k>l$ when $l \geq 2$, and if

$$
\left(f^{n} P(f)\right)^{(k)}\left(g^{n} P(g)\right)^{(k)}=p^{2}(z)
$$

where $P(w)=a_{m} w^{m}$ or $P(w)=c_{0}$ then $f(z)=b_{1} e^{b Q(z)}, g(z)=b_{2} e^{-b Q(z)}$, where $b_{1}, b_{2}$ and $c$ are three constants satisfying $a_{m}{ }^{2}\left(b_{1} b_{2}\right)^{n+m}((n+m) b)^{2}=-1$ or $c_{0}^{2}\left(b_{1} b_{2}\right)^{n}(n b)^{2}=-1$ and $Q(z)$ is a polynomial without constant such that $Q^{\prime}(z)=$ $p(z)$.

Proof. We first prove that

$$
f \neq 0, g \neq 0 \text {. }
$$

Let $P(w)=a_{m} w^{m}$. Then from (5) we get

$$
\left(f^{n+m}\right)^{(k)}\left(g^{n+m}\right)^{(k)}=p^{2}(z)
$$

Suppose that $z_{0}$ is a zero of $f$ say multiplicity $r$ but $p\left(z_{0}\right) \neq 0$ then $z_{0}$ is a pole of $g$ say multiplicity $s_{0}$. Then we have from (7), $(n+m) r-k=(n+m) s_{0}+k \Rightarrow(n+m)\left(r-s_{0}\right)=2 k$, which is contradiction for $n>2 k+2 l$. Now suppose that $z_{0}$ is a zero of $f$ say multiplicity $r_{1}$ if $z_{0}$ is not a pole of $g$ then $z_{0}$ must be zero of $p(z)$ of multiplicity $l_{0}$, say, then we have from (7) $(n+m) r_{1}-k>2 l_{0}$, which is again contradiction. If $z_{0}$ is a pole of $g$ say multiplicity $s_{1}$ then we have $(n+m) r_{1}-k=(n+m) s_{1}+k+2 l_{0},(n+m)\left(r_{1}-s_{1}\right)=2 k+2 l_{0}$ which is impossible. So, $f$ has no zeros. Similarly it can 
be shown that $g$ also has no zeros. Thus (6) is proved. Next we prove that

$$
\begin{aligned}
N(r, f) & =O(\log r) ; \\
N(r, g) & =O(\log r) ; \\
\left(f^{n} P(f)\right)^{(k)} & =\frac{p(z)^{2}}{\left(g^{n} P(g)\right)^{(k)}}
\end{aligned}
$$

Since $N\left(r,\left(f^{n} P(f)\right)(k)\right)=N\left(r, f^{n} P(f)\right)+k \bar{N}\left(r, f^{n} P(f)=(n+m) N(r, f)+k \bar{N}(r, f)+S(r, f)\right.$

By Lemma 5

$$
N\left(r, \frac{1}{\left(g^{n+m}\right)^{(k)}}\right) \leq N\left(r, \frac{1}{g^{n+m}}\right)+k \bar{N}\left(r, g^{n+m}\right)+O(\log r)=k \bar{N}(r, g)+O(\log r)
$$

using above inequality and (9) we get $(n+m) N(r, f)+k \bar{N}(r, f) \leq k \bar{N}(r, g)+O(\log r)$. Similarly we get $(n+m) N(r, g)+$ $k \bar{N}(r, g) \leq k \bar{N}(r, f)+O(\log r)$. Combining we get $N(r, f)+N(r, f)=O(\log r)$. Thus we obtain (8) which mean that $f$ and $g$ has at most finitely many poles. Now we prove that $\sigma(f)=\sigma(g)$. By K.Yamanoi [8] result of second fundamental theorem with taking $F=f^{n} P(f), G=g^{n} P(g)$ we get

$$
\begin{aligned}
T\left(r, F^{(k)}\right) & \leq \bar{N}\left(r, F^{(k)}\right)+\bar{N}\left(r, \frac{1}{F^{(k)}}\right)+\bar{N}\left(r, \frac{1}{F^{(k)}-p(z)}\right)+(\varepsilon+O(1)) T(r, F) \\
& \leq \bar{N}(r, f)+\bar{N}\left(r, \frac{1}{F^{(k)}}\right)+\bar{N}\left(r, \frac{1}{F^{(k)}-p(z)}\right)+(\varepsilon+O(1)) T(r, F)
\end{aligned}
$$

Therefore $T\left(r, F^{(k)}\right)-\bar{N}\left(r, \frac{1}{F^{(k)}}\right) \leq \bar{N}(r, f)+\bar{N}\left(r, \frac{1}{F^{(k)}-p(z)}\right)+(\varepsilon+O(1)) T(r, F)$ Using Lemma 4 we get, $T(r, F) \leq \bar{N}(r, f)+\bar{N}\left(r, \frac{1}{F^{(k)}-p(z)}\right)+N_{k+1}\left(r, \frac{1}{F}\right)+(\varepsilon+O(1)) T(r, F)$

$$
\begin{aligned}
(n+m) T(r, f) & \leq \bar{N}(r, f)+\bar{N}\left(r, \frac{1}{G^{(k)}-p(z)}\right)+(\varepsilon+O(1)) T(r, F) \\
& \leq \bar{N}(r, f)+(k+1)(n+m) T(r, g)+l \log r+(\varepsilon+O(1)) T(r, f)
\end{aligned}
$$

$\Rightarrow(n+m-l-1) T(r, f) \leq(k+1)(n+m) T(r, g)+(\varepsilon+O(1)) T(r, f)$

Since $\varepsilon<1$ then $T(r, f)=O(T(r, g))$,

Similarly, $T(r, g)=O(T(r, f))$ and hence

$$
\sigma(f)=\sigma(g)
$$

Then $f=\frac{e^{h(z)}}{r(z)}, g=\frac{e^{h_{1}(z)}}{q(z)}$ where $r(z)$ and $q(z)$ are polynomial with degree $\operatorname{deg}(r(z))=r, \operatorname{deg}(q(z))=q$, while $h(z)$ and $h_{1}(z)$ are nonconstant entire functions.

By Lemma 3, $h(z)$ and $h_{1}(z)$ are polynomial with $\operatorname{deg}(h(z))=\operatorname{deg}\left(h_{1}(z)\right)=h=\sigma(f)$, Then we have

$$
\left(f^{n+m}\right)^{(k)}=\frac{(m+n) e^{(n+m)(h(z))}}{r^{n+m+k}(z)} R_{k}(z)
$$

and

$$
\left(g^{n+m}\right)^{(k)}=\frac{(m+n) e^{(n+m)\left(h_{1}(z)\right)}}{q^{n+m+k}(z)} Q_{k}(z),
$$


where $R_{k}(z)$ and $Q_{k}(z)$ are two polynomials. From, (5) we get $h(z)+h_{1}(z)=C$, where $C$ is a constant. Furthermore we have $\operatorname{deg}\left(R_{k}(z)\right)+\operatorname{deg}\left(Q_{k}(z)\right)=\operatorname{deg}\left(r^{n+m+k}(z)\right)+\operatorname{deg}\left(q^{n+m+k}(z)\right)+2 l, \Rightarrow k(r+h-1)+k(q+h-1)=q(n+m+k)+$ $r(n+m+k)+2 l$.

$$
\Rightarrow 2 k(h-1)=(n+m)(q+r)+2 l
$$

If $N(r, f)+N(r, g) \neq 0$ then $(q+r) \geq 1$. From (11) we obtain $2 k(h-1) \geq(n+m)+2 l \Rightarrow n \leq 2 k(h-1)-(m+2 l)$ which contradict the given conditions. Therefore $N(r, f)+N(r, g)=0$, showing that both $f$ and $g$ are entire function and so $r=q=0$. From (11) we get $h=l+1, k=1$ or $h=2, k=l$

Case 1: For $k=1, h=l+1$. We get $h^{\prime}(z)=b p(z), h_{1}^{\prime}(z)=-b p(z)$ where $b \neq 0$ is a constant. $\Rightarrow h(z)=b Q(z)+d_{1}$ and $h_{1}(z)=-b Q(z)+d_{2}$, where $Q(z)$ is a polynomial without constant term such that $Q^{\prime}(z)=p(z)$ and $d_{1}, d_{2}$ are constants. Therefore $f=b_{1} e^{b Q(z)}, \quad g=b_{2} e^{-b Q(z)}$, where $b_{1}, \quad b_{2}$ are constant satisfying the condition $a_{m}^{2}\left(b_{1} b_{2}\right)^{n+m}((n+m) b)^{2}=-1$.

Case 2: $h=2, k=l$.

For this case we getting a contradiction by our assumption. The case $P(w)=c_{0}$ can be proved similarly. This completes the proof of the Lemma.

Lemma 9. [4] Let $f_{1}$ and $f_{2}$ be two nonconstant meromorphic functions. If $c_{1} f_{1}+c_{2} f_{2}=c_{3}$, where $c_{1}$, $c_{2}$, $c_{3}$ are nonzero constants, then

$$
T\left(r, f_{1}\right) \leq \bar{N}\left(r, f_{1}\right)+\bar{N}\left(r, \frac{1}{f_{1}}\right)+\bar{N}\left(r, \frac{1}{f_{2}}\right)+S\left(r, f_{1}\right)
$$

Lemma 10. Let $f$ and $g$ be two nonconstant meromorphic functions having zeros and poles of order at least s. Let $k, m, n$, be three integers with $n>\frac{2 k+1}{s}+m$ and let $P(w)=a_{m} w^{m}+a_{m-1} w^{m-1}+\cdots+a_{1} w+a_{0}$ or $P(w) \equiv c_{0}$, where $a_{0} \neq 0, a_{1}, \cdots, a_{m} \neq 0$ are complex constants. If $\left(f^{n} P(f)\right)^{(k)}=\left(g^{n} P(g)\right)^{(k)}$, then $f^{n} P(f)=g^{n} P(g)$.

Proof. From the assumption, we get $f^{n} P(f)=g^{n} P(g)+r(z)$ where $r(z)$ is a polynomial of degree at most $k-1$. If $r(z) \not \equiv 0$ then by Lemma 9 we have $T\left(r, \frac{f^{n} P(f)}{r(z)}\right) \leq \bar{N}\left(r, \frac{f^{n} P(f)}{r(z)}\right)+\bar{N}\left(r, \frac{r(z)}{f^{n} P(f)}\right)+\bar{N}\left(r, \frac{r(z)}{g^{n} P(g)}\right)+S(r, f)+S(r, g)$. Therefore,

$$
\begin{aligned}
T\left(r, f^{n} P(f)\right) & \leq T\left(r, \frac{f^{n} P(f)}{r(z)}\right)+(k-1) \log r+O(1) \\
& \leq \bar{N}\left(r, \frac{f^{n} P(f)}{r(z)}\right)+\bar{N}\left(r, \frac{r(z)}{f^{n} P(f)}\right)+\bar{N}\left(r, \frac{r(z)}{g^{n} P(g)}\right) \\
& +(k-1) \log r+S(r, f)+S(r, g) \\
& \leq \bar{N}(r, f)+\bar{N}\left(r, \frac{1}{f}\right)+\bar{N}\left(r, \frac{1}{g}\right)+\bar{N}\left(r, \frac{1}{P(f)}\right) \\
& +\bar{N}\left(r, \frac{1}{P(g)}\right)+2(k-1) \log r+S(r, f)+S(r, g)
\end{aligned}
$$

by Lemma 1 and $T(r, f) \geq s \log r+\mathrm{O}(1)$, we have

$$
(n+m) T(r, f) \leq\left(m+\frac{2}{s}+\frac{2(k-1)}{s}\right) T(r, f)+\left(m+\frac{1}{s}\right) T(r, g)+S(r, f)+S(r, g)
$$

Similarly, $(n+m) T(r, g) \leq\left(m+\frac{2}{s}+\frac{2(k-1)}{s}\right) T(r, g)+\left(m+\frac{1}{s}\right) T(r, f)+S(r, f)+S(r, g)$. By combining above two we get, $\left\{(n+m)-\left(2 m+\frac{2 k+1}{s}\right)\right\}(T(r, f)+T(r, g)) \leq S(r, f)+S(r, g)$, which is a contradiction. Hence $r(z) \equiv 0$ and so, $f^{n} P(f)=$ $g^{n} P(g)$. 
Lemma 11. [20] Let $f(z)$ and $g(z)$ be two nonconstant meromorphic functions, a be a finite nonzero constant. If $f$ and $g$ share a CM, then one of the following cases holds:

(i) $T(r, f) \leq N_{2}\left(r, \frac{1}{f}\right)+N_{2}\left(r, \frac{1}{g}\right)+N_{2}(r, f)+N_{2}(r, g)+S(r, f)+S(r, g)$; same holds for $T(r, g)$;

(ii) $f g=a^{2}$;

(iii) $f=g$.

Lemma 12. [1] Let $f(z)$ and $g(z)$ be two nonconstant meromorphic functions. If $f$ and $g$ share 1 IM and $H \not \equiv$, then

$$
T(r, f) \leq N_{2}\left(r, \frac{1}{f}\right)+N_{2}\left(r, \frac{1}{g}\right)+N_{2}(r, f)+N_{2}(r, g)+2 \bar{N}\left(r, \frac{1}{f}\right)+2 \bar{N}(r, f)+\bar{N}\left(r, \frac{1}{g}\right)+\bar{N}(r, g)+S(r, f)+S(r, g) .
$$

Lemma 13. [13] Let $f(z)$ and $g(z)$ be two nonconstant meromorphic functions, and $n(\geq 1), k(\geq 1), m(\geq 0)$ be three integers. Suppose that $F_{1}=\frac{\left(f^{n} P(f)\right)^{(k)}}{p(z)}$ and $G_{1}=\frac{\left(g^{n} P(g)\right)^{(k)}}{p(z)}$. If there exists two nonzero constants $c_{1}$ and $c_{2}$ such that $\bar{N}\left(r, \frac{1}{F-c_{1}}\right)=\bar{N}\left(r, \frac{1}{G_{1}}\right)$ and $\bar{N}\left(r, \frac{1}{G_{1}-c_{2}}\right)=\bar{N}\left(r, \frac{1}{F_{1}}\right)$, then $n \leq 3 k+m^{*}+3$, where $m^{*}= \begin{cases}m, & \text { if } P(f) \neq c_{0} ; \\ 0, & \text { if } P(f)=c_{0} ;\end{cases}$

\section{Proof of the main Theorems}

Proof of Theorem 15:

Proof. We discuss the following cases separately

Case (i): Let $P(w)=a_{m} w^{m}+a_{m-1} w^{m-1}+\cdots+a_{1} w+a_{0}$, where $a_{0}(\neq 0), a_{1}, \cdots, a_{m}(\neq 0)$ are constants, is not a monomials. Suppose that $F=\left(f^{n} P(f)\right)^{(k)}, G=\left(g^{n} P(g)\right)^{(k)}$ and $F^{*}=f^{n} P(f), G^{*}=g^{n} P(g)$ and also $F_{1}=\frac{F}{p(z)}$, $G_{1}=\frac{G}{p(z)}$. Since $F_{1}, G_{1}$ share $1 \mathrm{CM}$ by Lemma 11 one of the following subcases holds:

(a) $T\left(r, F_{1}\right) \leq N_{2}\left(r, \frac{1}{F_{1}}\right)+N_{2}\left(r, \frac{1}{G_{1}}\right)+N_{2}\left(r, F_{1}\right)+N_{2}\left(r, G_{1}\right)+S\left(r, F_{1}\right)+S\left(r, G_{1}\right)$ same holds for $T\left(r, G_{1}\right)$;

(b) $F_{1} G_{1}=p^{2}(z)$;

(c) $F_{1}=G_{1}$.

Subcase (a): We have

$$
T(r, F) \leq N_{2}\left(r, \frac{1}{F}\right)+N_{2}\left(r, \frac{1}{G}\right)+N_{2}(r, F)+N_{2}(r, G)+S(r, F)+S(r, G) .
$$

By Lemma 4 with $s=2$, we obtain

$$
\begin{aligned}
T\left(r, F^{*}\right) & \leq T(r, F)-N_{2}\left(r, \frac{1}{F}\right)+N_{k+2}\left(r, \frac{1}{F^{*}}\right)+S(r, F), \\
N_{2}\left(r, \frac{1}{G}\right) & \leq N_{k+2}\left(r, \frac{1}{G^{*}}\right)+k \bar{N}(r, G)+S(r, G) .
\end{aligned}
$$

Using (12), (14) in (13) we get,

$$
\begin{aligned}
T\left(r, F^{*}\right) & \leq N_{k+2}\left(r, \frac{1}{F^{*}}\right)+N_{k+2}\left(r, \frac{1}{G^{*}}\right)+(k+2) \bar{N}(r, g)+2 \bar{N}(r, f)+S(r, F)+S(r, G) \\
& \leq(k+2) \bar{N}\left(r, \frac{1}{f}\right)+N\left(r, \frac{1}{P(f)}\right)+(k+2) \bar{N}\left(r, \frac{1}{g}\right)+N\left(r, \frac{1}{P(g)}\right)+(k+2) \bar{N}(r, g)+2 \bar{N}(r, f)+S(r, f)+S(r, g) \\
& \leq \frac{k+2}{s} N\left(r, \frac{1}{f}\right)+N\left(r, \frac{1}{P(f)}\right)+\frac{k+2}{s} N\left(r, \frac{1}{g}\right)+N\left(r, \frac{1}{P(g)}\right)+\frac{k+2}{s} N(r, g)+\frac{2}{s} N(r, f)+S(r, f)+S(r, g)
\end{aligned}
$$


Now by first fundamental theorem and Lemma 1 we get

$$
(n+m) T(r, f) \leq\left(\frac{2 k+4}{s}+m\right) T(r, g)+\left(\frac{k+4}{s}+m\right) T(r, f)+S(r, f)+S(r, g) .
$$

Similarly,

$$
(n+m) T(r, g) \leq\left(\frac{2 k+4}{s}+m\right) T(r, f)+\left(\frac{k+4}{s}+m\right) T(r, g)+S(r, f)+S(r, g) .
$$

Combining above two inequality, we get

$$
\begin{aligned}
(n+m)\{T(r, f)+T(r, g)\} & \leq\left(\frac{3 k+8}{s}+2 m\right)\{T(r, f)+T(r, g)\}+S(r, f)+S(r, g) \\
& \Rightarrow\left\{n-\left(\frac{3 k+8}{s}+m\right)\right\}\{T(r, f)+T(r, g)\} \leq S(r, f)+S(r, g)
\end{aligned}
$$

which is a contradiction for $n>\left(\frac{3 k+8}{s}+m\right)$.

Subcase (b): Now by (b) we have

$$
\left(f^{n} P(f)\right)^{(k)} \cdot\left(g^{n} P(g)\right)^{(k)}=p^{2}(z),
$$

which is a contradiction by Lemma 7.

Subcase (c): By (c) we get

$$
\left(f^{n} P(f)\right)^{(k)}=\left(g^{n} P(g)\right)^{(k)} .
$$

Here $n>\left(\frac{3 k+8}{s}+m\right)>\left(\frac{2 k+1}{s}+m\right)$. So by Lemma 10 we get

$$
\begin{gathered}
f^{n} P(f)=g^{n} P(g) \\
\text { i.e., } f^{n}\left(a_{m} f^{m}+\ldots+a_{1} f+a_{0}\right)=g^{n}\left(a_{m} g^{m}+\ldots+a_{1} g+a_{0}\right) .
\end{gathered}
$$

Let $h=\frac{f}{g}$. If $h$ is a constant putting $f=g h$ in (15), we get

$$
a_{m} g^{n+m}\left(h^{n+m}-1\right)+a_{m-1} g^{n+m-1}\left(h^{n+m-1}-1\right)+\ldots \ldots+a_{0} g^{n}\left(h^{n}-1\right)=0
$$

which implies $h^{d}=1$, where $d=\operatorname{gcd}(n+m, n+m-1, \ldots, n+m-i, \ldots, n+1, n), a_{m-i} \neq 0$ for some $i \in\{0,1, \ldots, m\}$. Thus $f=\operatorname{tg}$ for some $t$ such that $t^{d}=1$, where $d=\operatorname{gcd}(n+m, n+m-1, \ldots, n+m-i, \ldots, n+1, n), a_{m-i} \neq 0$ for some $i \in\{0,1, \ldots, m\}$. If $h$ is not a constant then from (15) we see $f$ and $g$ satisfy the algebraic equation $R(f, g)=0$ where $R(f, g)$ is given by (1)

Case (ii): When $P(w)=a_{m} w^{m}$, or $P(w)=c_{0} a_{m} \neq 0, c_{0}$ are complex constant. Proceeding as in case (i) above we obtain $F_{1} G_{1}=1$ or $F_{1}=G_{1}$.

If $F_{1} G_{1}=1$ then by Lemma 8 gives $f(z)=b_{1} e^{b Q(z)}, g(z)=b_{2} e^{-b Q(z)}$, where $Q(z)$ is a polynomial without constant such that $Q^{\prime}(z)=p(z), b_{1}, b_{2}$ and $b$ are three constants satisfying $a_{m}^{2}\left(b_{1} b_{2}\right)^{n+m}((n+m) b)^{2}=-1$ or $c_{0}^{2}\left(b_{1} b_{2}\right)^{n}(n b)^{2}=-1$. If $F_{1}=G_{1}$ we obtain $f=\operatorname{tg}$ for a constant $t$ such that $t^{n+m^{*}}=1$.

Proof of Theorem 16:

Proof. Case (i): Let $P(w)$ is not a monomial. Suppose that $F=\left(f^{n} P(f)\right)^{(k)}, G=\left(g^{n} P(g)\right)^{(k)}$ and $F^{*}=f^{n} P(f), G^{*}=$ $g^{n} P(g)$ and also $F_{1}=\frac{F}{p(z)}, G_{1}=\frac{G}{p(z)}$. Then $F_{1}, G_{1}$ share $1 \mathrm{IM}$. We assume that $H \not \equiv 0$ defined as in (2). So, from Lemma 
12 we have

$$
\begin{aligned}
T\left(r, F_{1}\right) & \leq N_{2}\left(r, \frac{1}{F_{1}}\right)+N_{2}\left(r, \frac{1}{G_{1}}\right)+N_{2}\left(r, F_{1}\right)+N_{2}\left(r, G_{1}\right)+2 \bar{N}\left(r, \frac{1}{F_{1}}\right) \\
& +2 \bar{N}\left(r, F_{1}\right)+\bar{N}\left(r, \frac{1}{G_{1}}\right)+\bar{N}\left(r, G_{1}\right)+S(r, f)+S(r, g) .
\end{aligned}
$$

i;e.,

$$
\begin{aligned}
T(r, F) & \leq N_{2}\left(r, \frac{1}{F}\right)+N_{2}\left(r, \frac{1}{G}\right)+N_{2}(r, F)+N_{2}(r, G)+2 \bar{N}\left(r, \frac{1}{F}\right) \\
& +\bar{N}\left(r, \frac{1}{G}\right)+2 \bar{N}(r, F)+\bar{N}(r, G)+S(r, f)+S(r, g) .
\end{aligned}
$$

Now by Lemma 4 with $s=2$, we get

$$
T\left(r, F^{*}\right) \leq T(r, F)-N_{2}\left(r, \frac{1}{F}\right)+N_{2+k}\left(r, \frac{1}{F^{*}}\right)+S(r, f) .
$$

and

$$
N_{2}\left(r, \frac{1}{G}\right) \leq k \bar{N}(r, G)+N_{2+k}\left(r, \frac{1}{G^{*}}\right)+S(r, g) .
$$

Using (16), (18) in (17) we have,

$$
\begin{aligned}
T\left(r, F^{*}\right) & \leq N_{2+k}\left(r, \frac{1}{F^{*}}\right)+N_{2}\left(r, \frac{1}{G}\right)+N_{2}(r, F)+N_{2}(r, G)+2 \bar{N}\left(r, \frac{1}{F}\right)+\bar{N}\left(r, \frac{1}{G}\right)+2 \bar{N}(r, F)+\bar{N}(r, G)+S(r, f)+S(r, g) \\
& \leq N_{2+k}\left(r, \frac{1}{F^{*}}\right)+N_{2+k}\left(r, \frac{1}{G^{*}}\right)+k \bar{N}(r, G)+N_{2}(r, F)+N_{2}(r, G)+2 \bar{N}\left(r, \frac{1}{F}\right) \\
& +\bar{N}\left(r, \frac{1}{G}\right)+2 \bar{N}(r, F)+\bar{N}(r, G)+S(r, f)+S(r, g) \\
& \leq(3 k+4) \bar{N}\left(r, \frac{1}{f}\right)+3 N\left(r, \frac{1}{P(f)}\right)+(2 k+3) \bar{N}\left(r, \frac{1}{g}\right) \\
& +2 N\left(r, \frac{1}{P(g)}\right)+(2 k+3) \bar{N}(r, g)+(2 k+4) \bar{N}(r, f)+S(r, f)+S(r, g)
\end{aligned}
$$

By using Lemma 1 and 1st fundamental theorem, we get

$$
\begin{aligned}
(n+m) T(r, f) & \leq \frac{3 k+4}{s} T(r, f)+3 m T(r, f)+\frac{2 k+3}{s} T(r, g)+2 m T(r, g)+\frac{2 k+3}{s} T(r, g)+\frac{2 k+4}{s} T(r, f)+S(r, f)+S(r, g) \\
& \leq\left(\frac{5 k+8}{s}+3 m\right) T(r, f)+\left(\frac{4 k+6}{s}+2 m\right) T(r, g)+S(r, f)+S(r, g)
\end{aligned}
$$

Similarly,

$$
(n+m) T(r, g) \leq\left(\frac{5 k+8}{s}+3 m\right) T(r, g)+\left(\frac{4 k+6}{s}+2 m\right) T(r, f)+S(r, f)+S(r, g)
$$

Combining (19) and (20) we get

$$
\left\{n-\left(\frac{9 k+14}{s}+4 m\right)\right\}(T(r, f)+T(r, g)) \leq S(r, f)+S(r, g)
$$


which is a contradiction as $n>\max \left\{2 k+2 l, \frac{9 k+14}{s}+4 m, 2 k(\sigma(f)-1)-(m+2 l)\right\}$. Therefore $H \equiv 0$. This gives,

$$
\left(\frac{F_{1}^{(2)}}{F_{1}^{(1)}}-2 \frac{F_{1}^{(1)}}{F_{1}-1}\right)=\left(\frac{G_{1}^{(2)}}{G_{1}^{(1)}}-2 \frac{G_{1}^{(1)}}{G_{1}-1}\right) .
$$

Integrating both sides of the above equality twice we get

$$
\Rightarrow \frac{1}{F_{1}-1}=\frac{A}{G_{1}-1}+B
$$

where $A \neq 0, B$ are constants. We now discuss the following three subcases:

Subcase (i): Let $B \neq 0$ and $A=B$. Then from (22) we get

$$
\Rightarrow \frac{1}{F_{1}-1}=\frac{B G_{1}}{G_{1}-1} .
$$

If $B=-1$ then from above equation we get

$$
F_{1} G_{1}=1
$$

i;e.,

$$
\left(f^{n} P(f)\right)^{(k)} \cdot\left(g^{n} P(g)\right)^{(k)}=p^{2}(z) .
$$

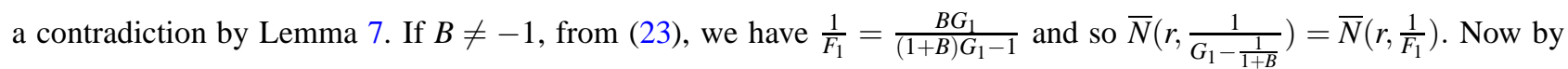
Nevanlinna second fundamental theorem, we get

$$
T\left(r, G_{1}\right) \leq \bar{N}\left(r, \frac{1}{G_{1}}\right)+\bar{N}\left(r, \frac{1}{G_{1}-\frac{1}{1+B}}\right)+\bar{N}\left(r, G_{1}\right)+S\left(r, G_{1}\right) \leq \bar{N}\left(r, \frac{1}{G_{1}}\right)+\bar{N}\left(r, \frac{1}{F_{1}}\right)+\bar{N}\left(r, G_{1}\right)+S\left(r, G_{1}\right) .
$$

Using Lemma 4

$$
T(r, G) \leq N_{k+1}\left(r, \frac{1}{f^{n} P(f)}\right)+k \bar{N}(r, f)+T(r, G)+N_{k+1}\left(r, \frac{1}{g^{n} P(g)}\right)-(n+m) T(r, g)+\bar{N}(r, g)+S(r, g) .
$$

Therefore,

$$
\begin{aligned}
& (n+m) T(r, g) \leq(k+1) \bar{N}\left(r, \frac{1}{f}\right)+N\left(r, \frac{1}{P(f)}\right)+k \bar{N}(r, f)+(k+1) \bar{N}\left(r, \frac{1}{g}\right)+N\left(r, \frac{1}{P(g)}\right)+\bar{N}(r, g)+S(r, f)+S(r, g) \\
& (n+m) T(r, g) \leq\left(\frac{2 k+1}{s}+m\right) T(r, f)+\left(\frac{k+2}{s}+m\right) T(r, g)+S(r, f)+S(r, g) .
\end{aligned}
$$

Similarly,

$$
(n+m) T(r, f) \leq\left(\frac{2 k+1}{s}+m\right) T(r, g)+\left(\frac{k+2}{s}+m\right) T(r, f)+S(r, f)+S(r, g) .
$$

Combining above two inequality, we get

$$
\left(n-\left(\frac{3 k+3}{s}+m\right)\right)\{T(r, f)+T(r, g)\} \leq S(r, f)+S(r, g) .
$$

Which contradict our assumption.

Subcase (ii): 
Let $B \neq 0$ and $A \neq B$. Then from (22) we get $F_{1}=\frac{(B+1) G_{1}-(B-A+1)}{B G_{1}+(A-B)}$ and so $\bar{N}\left(r, \frac{1}{G_{1}-\frac{B-A+1}{B+1}}\right)=\bar{N}\left(r, \frac{1}{F_{1}}\right)$. Proceeding as in subcase (i) we get a contradiction.

Subcase (iii): Let $B=0$ and $A \neq 0$. Then (22) gives $F_{1}=\frac{G_{1}+A-1}{A}$ and $G_{1}=A F_{1}-(A-1)$. If $A \neq 1$, we have $\bar{N}\left(r, \frac{1}{F_{1}-\frac{A-1}{A}}\right)=\bar{N}\left(r, \frac{1}{G}\right)$ and $\bar{N}\left(r, \frac{1}{G_{1}-(1-A)}\right)=\bar{N}\left(r, \frac{1}{F_{1}}\right)$. Using the Lemma 13 we have $n \leq 3 k+m+3$, a contradiction. Thus $A=1$ and hence $F_{1}=G_{1}$

$$
\left(f^{n} P(f)\right)^{(k)}=\left(g^{n} P(g)\right)^{(k)} .
$$

Here $n>\left(\frac{9 k+14}{s}+4 m\right)>\left(\frac{2 k+1}{s}+m\right)$. So by Lemma 10 we get

$$
\begin{gathered}
f^{n} P(f)=g^{n} P(g), \\
\text { i.e., } f^{n}\left(a_{m} f^{m}+\ldots+a_{1} f+a_{0}\right)=g^{n}\left(a_{m} g^{m}+\ldots+a_{1} g+a_{0}\right) .
\end{gathered}
$$

Let $h=\frac{f}{g}$.

If $h$ is a constant putting $f=g h$ in (24), we get

$$
a_{m} g^{n+m}\left(h^{n+m}-1\right)+a_{m-1} g^{n+m-1}\left(h^{n+m-1}-1\right)+\ldots \ldots+a_{0} g^{n}\left(h^{n}-1\right)=0
$$

which implies $h^{d}=1$, where $d=\operatorname{gcd}(n+m, n+m-1, \ldots, n+m-i, \ldots, n+1, n), a_{m-i} \neq 0$ for some $i \in\{0,1, \ldots, m\}$. Thus $f=t g$ for some $t$ such that $t^{d}=1$, where $d=\operatorname{gcd}(n+m, n+m-1, \ldots, n+m-i, \ldots, n+1, n), a_{m-i} \neq 0$ for some $i \in\{0,1, \ldots, m\}$. If $h$ is not a constant then from (24) we see $f$ and $g$ satisfy the algebraic equation $R(f, g)=0$ where $R(f, g)$ is given by (1).

Case (ii): When $P(w)=a_{m} w^{m}$, or $P(w)=c_{0} a_{m} \neq 0, c_{0}$ are complex constant. Proceeding as in case (i) above we obtain $F_{1} G_{1}=1$ or $F_{1}=G_{1}$. If $F_{1} G_{1}=1$ then by Lemma 8 gives $f(z)=b_{1} e^{b Q(z)}, g(z)=b_{2} e^{-b Q(z)}$, where $Q(z)$ is a polynomial without constant such that $Q^{\prime}(z)=p(z), b_{1}, b_{2}$ and $b$ are three constants satisfying $a_{m}{ }^{2}\left(b_{1} b_{2}\right)^{n+m}((n+m) b)^{2}=-1$ or $c_{0}^{2}\left(b_{1} b_{2}\right)^{n}(n b)^{2}=-1$. If $F_{1}=G_{1}$ we obtain $f=t g$ for a constant $t$ such that $t^{n+m^{*}}=1$

Following example is the supportive example of Theorem 15, when the polynomial $P(w)$ is not a monomial.

Example 1. Let $P(w)=a_{4} w^{4}+a_{0}$, where $a_{0}, a_{4} \in \mathbb{C} \backslash\{0\}$ and $p(z)=z^{3}-3 z^{2}+z-1$. Let

$$
f(z)=\frac{\left(e^{z}-a\right)^{2}}{\left(e^{z}-b\right)^{3}} \text { and } g(z)=-i \frac{\left(e^{z}-a\right)^{2}}{\left(e^{z}-b\right)^{3}}
$$

where $a, b \in \mathbb{C} \backslash\{0\}$ with $a \neq b$. Let $k=4, n=16$. Clearly $s=2$. Now

$$
f^{16} P(f)=\frac{\left(e^{z}-a\right)^{32}}{\left(e^{z}-b\right)^{60}}\left\{a_{4}\left(e^{z}-a\right)^{8}+a_{0}\left(e^{z}-b\right)^{12}\right\}
$$

and

$$
g^{16} P(g)=\frac{\left(e^{z}-a\right)^{32}}{\left(e^{z}-b\right)^{60}}\left\{a_{4}\left(e^{z}-a\right)^{8}+a_{0}\left(e^{z}-b\right)^{12}\right\}
$$


Thus we see that $f$ and $g$ are two non-constant meromorphic functions having zeros and poles of multiplicity at least 2 , and $\left[f^{16} P(f)\right]^{(4)}$ and $\left[g^{16} P(g)\right]^{(4)}$ share the polynomial $p(z) C M$ with

$$
n>\max \left\{2 k+2 l, \frac{3 k+8}{s}+m^{*}, 2 k(\sigma(f)-1)-(m+2 l)\right\} .
$$

We thus see that one of the conclusion $f \equiv \operatorname{tg}$ of Theorem 15 holds good where $t^{d}=(-i)^{\operatorname{gcd}(20,16)}=(-i)^{4}=1$.

The next example is the supportive example of Theorem 15 when the polynomial $P(w)$ is a monomial.

Example 2. Let $f=\tan z$, and $g(z)=-\tan z, p(z)=a_{2} z^{2}+a_{0}$, where $a_{0}, a_{2} \in \mathbb{C} \backslash\{0\}, P(w)=w^{2}$. Let $n=18$. Clearly $s=1, l=2, m^{*}=2$ and

$$
n>\max \left\{2 k+2 l, \frac{3 k+8}{s}+m^{*}, 2 k(\sigma(f)-1)-(m+2 l)\right\}
$$

We also see that $\left[f^{18} P(f)\right]^{(2)}=\left[f^{20}\right]^{(2)}=\left[\tan ^{20} z\right]^{(2)}$ and $\left[g^{18} P(g)\right]^{(2)}=\left[g^{20}\right]^{(2)}=\left[\tan ^{20} z\right]^{(2)}$ share the polynomial $p(z)$ $C M$, and one of the conclusion $f \equiv \operatorname{tg}$ of Theorem 15 holds good where $t^{d}=(-1)^{\operatorname{gcd}(20,18)}=(-1)^{2}=1$.

The next example is the supportive example of Theorem 15 when the polynomial $P(w)=c_{0}$.

\section{Example 3.Let}

$$
f(z)=\frac{2+3 i}{1-5 i} e^{3 z^{3}+2 z^{2}-z+6} \text { and } g(z)=\frac{1-5 i}{2+3 i} e^{-\left(3 z^{3}+2 z^{2}-z+6\right)}
$$

Let $n=13, P(w)=c_{0}=\frac{i}{13}$ and $k=1$. Here we see that $b_{1}=\frac{2+3 i}{1-5 i}$ and $b_{2}=\frac{1-5 i}{2+3 i}$ and $b=1$. It is clear that

$$
c_{0}^{2}\left(b_{1} b_{2}\right)^{n}(n b)^{2}=-1 \text {. }
$$

Let $Q(z)=3 z^{3}+2 z^{2}-z+6$ and $p(z)=9 z^{2}+4 z-1$. Clearly, $Q^{\prime}(z)=p(z)$. Clearly

$$
n>\max \left\{2 k+2 l, \frac{3 k+8}{s}+m^{*}, 2 k(\sigma(f)-1)-(m+2 l)\right\}
$$

is satisfied. We see that

$$
\begin{aligned}
\left(f^{13} P(f)\right)^{\prime} & =\frac{i}{13}\left(\frac{2+3 i}{1-5 i}\right)^{13}\left(e^{13\left(3 z^{3}+2 z^{2}-z+6\right)}\right)^{\prime} \\
& =\frac{i}{13}\left(\frac{2+3 i}{1-5 i}\right)^{13} \cdot 13\left(9 z^{2}+4 z-1\right) \cdot e^{13\left(3 z^{3}+2 z^{2}-z+6\right)} \\
& =i\left(\frac{2+3 i}{1-5 i}\right)^{13} \cdot p(z) e^{13\left(3 z^{3}+2 z^{2}-z+6\right)}
\end{aligned}
$$

Similarly,

$$
\left(g^{13} P(g)\right)^{\prime}=-i\left(\frac{1-5 i}{2+3 i}\right)^{13} \cdot p(z) e^{-13\left(3 z^{3}+2 z^{2}-z+6\right)}
$$

Clearly $\left(f^{13} P(f)\right)^{\prime}$ and $\left(g^{13} P(g)\right)^{\prime}$ share the polynomial $p(z) C M$. We see that $f$ and $g$ are of the forms $f(z)=b_{1} e^{b Q(z)}$ and $g(z)=b_{2} e^{-b Q(z)}$ with $c_{0}^{2}\left(b_{1} b_{2}\right)^{n}(n b)^{2}=-1$. 


\section{Acknowledgement}

The authors would like to thanks the reviewers for their valuable comments and suggestions.

\section{Competing interests}

The authors declare that they have no competing interests.

\section{Authors' contributions}

All authors have contributed to all parts of the article. All authors read and approved the final manuscript.

\section{References}

[1] A. Banerjee, Meromorphic functions sharing one value, Int. J. Math. Math. Sci. 22 (2005), 3587-3598.

[2] C. C. Yang, On deficiencies of differential polynomials II, Math. Z. 125 (1972), 107-112.

[3] H. H. Chen, M. L. Fang, On the value distrbution of $f^{n} f^{\prime}$, Sci. China Ser. A 38 (1995), 789-798.

[4] H. X. Yi, C. C. Yang, Uniqueness theory of meromorphic functions(in Chinese), Science Press, Beijing, 1995.

[5] I. Lahiri, S. Dewan, Value distribution of the product of a meromorphic functions and its derivative, Kodai Math. J. 26 (2003), 95-100.

[6] J. L. Zhang, Uniqueness theorems for entire functions concerning fixed points, Comput. Math. Appl. 56 (2008), 3079-3087.

[7] J. F Xu, F. Lï, H. X. Yi, Fixed points and uniqueness of meromorphic functions, Comput. Math. Appl. 59 (2008), 9-17.

[8] K. Yamanoi, The second main theorem for small functions and related problems, Acta Math. 192 (2004), $225-294$.

[9] L. Yang, Value distributions theory, Springer-Verlag, Berlin, 1993.

[10] M. L. Fang, H. L. Qiu, Meromorphic functions that share fixed points, J. Math. Anal. Appl. 268 (2002), 426-439.

[11] M. L. Fang, Uniqueness and value sharing of entire functions, Comput. Math. Appl. 44 (2002), 828-831.

[12] M. L. Fang, X. H. Hua, Entire functions that share one value, Nanjing Univ. J. Math. Biquarterly 13 (1996), 44-48.

[13] P. Sahoo, Meromorphic functions that share fixed points with finite weights, Bull. Math. Anal. Appl. 2 (2010), 106-118.

[14] P. Sahoo, Uniqueness and differential polynomials of meromorphic functions sharing a polynomial, Pales. J. Math. 5 (2016), $46-56$.

[15] W. Bergweiler, A. Eremenko, On the singularities of the inverse to a meromorphic function of finite order, Rev. Mat. Iberoam. 11 (1995), 355-373.

[16] W. C. Lin, H. X. Yi, Uniqueness theorems for meromorphic functions concerning fixed points, Complex Var. Theory Appl. 49 (2004), 793-806.

[17] W. C. Lin, H. X. Yi, Uniqueness theorems for meromorphic functions, Indian J. Pure Appl. Math. 35 (2004), 121-132.

[18] W. K. Hayman, Meromorphic function, Clarendon Press, Oxford, 1964.

[19] X. B. Zhang, J. F. Xu, Uniqueness of meromorphic functions sharing a small function and its applications, compt. Math. Appl. 61 (2011), 722-730.

[20] X. H. Hua, C. C. Yang, Uniqueness and value-sharing of meromorphic functions, Ann. Acad. Sci. Fenn. Math. 22 (1997), $395-406$. 